\title{
Calcium and phosphorus co-doped carbon dots enhance osteogenic differentiation for calvarial defect repair in situ
}

\section{Lin Wu}

Nanjing Medical University

\section{Yunchao Wu}

Third Affiliated Hospital of Changzhou

Jingjin Liu

Jiangsu University

Suwan Liu

Jiangsu University

Qianzhe Li

Jiangsu University

Mengyu Yang

Jiangsu University

\section{Miaomiao Zhang}

Jiangsu University

\section{Qing Ji}

Jiangsu University

Feimin Zhang

Nanjing Medical University

Fengyi Du ( $\sim$ biodfy@ujs.edu.cn )

Jiangsu University https://orcid.org/0000-0002-3555-421X

\section{Nano Express}

Keywords: Carbon dots, Calcium, Phosphorus, Calvarial defect

Posted Date: July 12th, 2021

DOI: https://doi.org/10.21203/rs.3.rs-698683/v1

License: (c) (1) This work is licensed under a Creative Commons Attribution 4.0 International License.

Read Full License 


\section{Abstract}

Calvarial bone defect remains a clinical challenge due to the lack of efficient osteo-inductive agent. Herein, a novel calcium and phosphorus codoped carbon dot (Ca/P-CD) for bone regeneration was synthesized using phosphoethanolamine and calcium gluconate as precursors. The resultant $\mathrm{Ca} / \mathrm{P}-\mathrm{CDs}$ exhibited ultra-small size, stable excitation dependent emission spectra and favorable dispersibility in water. Moreover, $\mathrm{Ca} / \mathrm{P}-\mathrm{CD}$ s with good biocompatibility rapidly entered the cytoplasm through endocytosis and increased the expression of bone differentiation genes. After mixing with temperature-sensitive hydrogel, $\mathrm{Ca} / \mathrm{P}-\mathrm{CD}$ s were injected in situ into calvarial defect and promoted the repair of bone injury. These $\mathrm{Ca} / \mathrm{P}-\mathrm{CD}$ s provide a new treatment method for the bone repair and expend the application in the biomedical fields.

\section{Introduction}

Calvarial bone defect represents a frequent pathological issue of orthopedic which can be caused by trauma, congenital malformations, infections, and surgery ${ }^{[1-3]}$. In clinic, the therapeutic effect of calvarial bone defect is unsatisfactory owing to the lack of efficient osteo-inductive agent. In addition, traditional surgical transplantation has serious side effects and limited application including graft selection and immune rejection ${ }^{[4,5]}$. Therefore, there is an urgent need to develop effective treatment strategy for promoting bone regeneration effectively and restoring bone function.

It is well-known that calcium and phosphorus are important elements in the process of maintaining normal bone differentiation and development. They cooperate with parathyroid hormone (PTH) and calcitonin (CT) to promote the expression of genes related to bone differentiation, and achieve the regulation of bone cell function. In addition, both of elements have an impact on bone metabolism and bone tissue morphology ${ }^{[6]}$. For instance, hydroxyapatite $(\mathrm{HA})$ with a general formula of $\mathrm{Ca}_{10}(\mathrm{OH})_{2}\left(\mathrm{PO}_{4}\right)_{6}$ has extensively applied in bone defect repair due to its chemical similarities with the natural bone ${ }^{[7,8]}$. However, the uncontrollability of morphology and metabolism in vivo of HA weakens its therapeutic effect. As a new type of nano-biomaterial, carbon dots (CDs) have a ultra-small size (2-8nm), excellent optical properties, good biological safety and stability $[9,10]$. It has attracted widespread attention in the fields of bioimaging, immunolabeling, molecular tracing, tumor targeting, and drug delivery ${ }^{[11]}$. By modifying the abundant active groups on the surface, CDs can be endowed with different specific functions to meet specific biomedical needs ${ }^{[12]}$. Our research group also has reported a series of heteroatomic doped CDs that possessed distinct functions ${ }^{[13-15]}$.

The focus of this research is to synthesize functionalized CDs with calcium and phosphorus doping for bone regeneration and repair of bone injury. The physical and chemical properties of $\mathrm{Ca} / \mathrm{P}-\mathrm{CD}$ s was characterized via a series of methods. Afterwards, the function of osteogenesis induced by $\mathrm{Ca} / \mathrm{P}-\mathrm{CDs}$ was explored using mouse osteoblastic cell line (MC3T3-E1) in vitro. Meanwhile, Ca/P-CDs mixed with thermo-sensitive hydrogel (Pluronic F127) were injected into calvarial defect in mice to investigate the 
bone regeneration in situ. Finally, we verified the long-term biocompatibility of $\mathrm{Ca} / \mathrm{P}-\mathrm{CD}$ s through histologic section.

\section{Material And Method \\ 2.1 Materials}

Phosphoethanolamine, calcium gluconate, diethylenetriamine pentaacetic acid (DTPA), and glycine were purchased from Aladdin Reagent Company (Shanghai, China). Fetal bovine serum and Dulbecco's minimum essential medium (DMEM) were procured from Hyclone (Logan, UT, USA). Lyso tracker and Mito tracker were purchased from Beyotime Biotechnology (Beijing, China). qPCR kits were purchased from Sigma (NY, USA). ICR mice were purchased from Center for Experimental Animals of Jiangsu University. Recombinant Human Bone Morphogenetic Protein-2 (rhBMP-2) was obtained from R\&D Systems Europe (United Kingdom). All of these chemical agents were of analytical grade and were utilized without further purification.

\subsection{Synthesis of P-CDs, Ca-CDs and $\mathrm{Ca} / \mathrm{P}-\mathrm{CDs}$}

Three types of CDs were synthesized using different precursors by a one-pot hydrothermal method, as described in the literature previously with a little modification ${ }^{[16]}$. P-CDs: $0.4 \mathrm{~g}$ of phosphoethanolamine and $0.40 \mathrm{~g}$ of DTPA were dissolved in $20 \mathrm{ml}$ of double-steaming water and stirred continuously for $10 \mathrm{~min}$ to form a transparent solution. The solution was transferred to a reaction kettle and heated in a muffle furnace at $200^{\circ} \mathrm{C}$ for $4 \mathrm{~h}$. After the system was cooled to room temperature naturally, the liquid was poured into a $50 \mathrm{ml}$ centrifugal tube and the black impurities are removed by centrifuging at $2000 \mathrm{rpm}$ for $15 \mathrm{~min}$. The prepared solution was then dialyzed against water for 5 days in a cut-off dialysis bag ( $M W C O=3$ kD, Solarbio Company, Beijing, China). The dialysate was collected and freeze-dried with vacuum freeze dryer. Thus, P-CDs powder was obtained and stored for further characterization. The Ca$\mathrm{CDs}$ and $\mathrm{Ca} / \mathrm{P}-\mathrm{CD}$ s were prepared using calcium gluconate and DTPA, and phosphoethanolamine and calcium gluconate as precursors, respectively.

\subsection{Morphological and chemical characterization of $\mathrm{Ca} / \mathrm{P}$ - CDs}

The morphologies of the Ca/P-CDs were observed by high-resolution transmission electron microscopy (HRTEM) on a JEM-2100 microscope (JEOL, Tokyo, Japan) under an accelerating voltage of $200 \mathrm{kV}$. Elemental composition of the Ca/P-CDs was determined by X-ray photoelectron spectroscopy (XPS) on Escalab 250Xi (Thermo Scientific, America). The surface chemical components of $\mathrm{Ca} / \mathrm{P}$-CDs were examined using a Fourier transform infrared (FT-IR) spectrometer (Nicolet Nexus 470; GMI, Franklin, IN, USA) ranged from 4000 to $400 \mathrm{~cm}^{-1}$. The crystal structure of Ca/P-CDs was analyzed by $x$-ray diffraction (XRD) on a Rigaku-D/MAX2500 diffractometer (Japan) with a scanning speed of $4 \% \mathrm{~min}$ in the range from $5-90^{\circ}$. The optical properties of the Ca/P-CDs were obtained with a UV-2450 UV/vis 
spectrophotometer (Shimadzu, Japan), and the photoluminescence emission spectra was recorded using a Cary Eclipse Fluorometer (Varian, Palo Alto, CA, USA).

\subsection{Cell culture and biocompability of $\mathrm{Ca} / \mathrm{P}-\mathrm{CDs}$}

In vitro cytotoxicity of the Ca/P-CDs was determined using the CCK-8 cell viability kit assay ( Solarbio, Beijing, China). Briefly, MC3T3-E1 (mouse embryo osteoblast precursor) cells ( $1 \times 10^{4}$ cells per well) were seeded into a 96-well plate with four replicates in each group. After incubation at $37^{\circ} \mathrm{C}$ and $5 \% \mathrm{CO}_{2}$ for 24 $\mathrm{h}$, the different concentrations of $\mathrm{Ca} / \mathrm{P}-\mathrm{CDs}(50 \mu \mathrm{g} / \mathrm{mL})$ in fresh DMEM were used to replace the growth medium and incubated for another $24 \mathrm{~h}$. Then, the cells were washed with PBS, and $10 \mu \mathrm{L} \mathrm{CCK}-8$ and $90 \mu \mathrm{l}$ DMEM solution were added to each well. Next, the plates were incubated for $4 \mathrm{~h}$ at $37^{\circ} \mathrm{C}$ and $5 \%$ $\mathrm{CO} 2$. Finally, the absorbance of each well was detected at the emission wavelength of $450 \mathrm{~nm}$ using a Synergy HT Multi- Mode Microplate Reader (Bio Tek, Winooski, VT, USA). Nontreated cells (in DMEM) were used as a control, and the relative cell viability (mean $\pm S D, n=3$ ) was expressed as (Abs sample - Abs zero sitting)/(Abscontrol-Abszero sitting) $\times 100 \%$. The experiment was repeated three times independently.

\subsection{Hemolysis assay}

All animal procedures were conducted in accordance with the Management Rules of the Ministry of Health of the People's Republic of China and approved by the Institutional Animal Care and Use Committee of Jiangsu University (permit number: SYXK2018-053). The hemocompatibility of Ca/P-CDs was carried out according to the protocol reported in the literature with slight modification ${ }^{[13]}$. In brief, fresh mouse blood was stabilized with heparin sodium and centrifuged (1, $200 \mathrm{rpm}, 15 \mathrm{~min})$ to remove the supernatant. The sediment was washed with PBS five times to obtain the mouse red blood cells (MRBCs). Next, the mouse RBCs were resuspended using $0.9 \mathrm{~mL}$ PBS containing Gd-doped CDs with different particle concentrations from 50 to $200 \mathrm{mg} / \mathrm{mL}$ (water as positive control and PBS as negative control). These samples were incubated for $2 \mathrm{~h}$ at $37^{\circ} \mathrm{C}$ after gentle shaking, thus centrifuged to collect the supernatant at 12,000 rpm for $1 \mathrm{~min}$. The absorbance of samples at $541 \mathrm{~nm}$ was recorded by a UVVis spectrophotometer (UV-2450). The hemolysis percentages of Ca/P-CDs was calculated using the absorbance compared with the control.

\subsection{Cytophagocytosis assay}

The internalization of Ca/P-CDs in the cells was observed using laser confocal microscope. The MC3T3E1 cells were seeded into the 24-well plates that pre-filled with $10 \mathrm{~mm}$ coverslips. When the cell density reached $60 \%$, the MC3T3-E1 cells were administered with the medium containing $200 \mu \mathrm{g} / \mathrm{ml}$ of Ca/P-CDs and further incubated for $4 \mathrm{~h}$. Then, these MC3T3-E1 cells on the coverslips were washed with phosphatebuffered saline (PBS) twice and fixed with $4 \%$ paraformaldehyde (PFA). In order to determine the intracellular distribution, these MC3T3-E1 cells stained with Lyso tracker and Mito tracker were observed under a confocal laser scanning fluorescence microscope (Zeiss LSM-710, Carl Zeiss Meditec AG, Jena, Germany) 


\subsection{Alizarin red staining}

Alizarin red staining was choosed to verify the osteogenic differentiation of MC3T3-E1 cells after incubation with Ca/P-CDs. MC3T3-E1 cells were seeded into a 96-well plate at a density of $1 \times 10^{4}$ cells per well with four replicates. After incubation with $50 \mu \mathrm{g} / \mathrm{mL} \mathrm{Ca/P-CDs} \mathrm{for} 21$ days, the MC3T3-E1 cells were washed with PBS and fixed with PBS paraformaldehyde (3.6 \%) for 1 hour at $4^{\circ} \mathrm{C}$. Then, the MC3T3-E1 cells were stained with $1 \%$ alizarin red (sigma Aldrich) solution for 30 minutes. Finally, these MC3T3-E1 cells were washed by PBS to remove free alizarin red, and the $\mathrm{Ca} / \mathrm{P}-\mathrm{CD}$-induced mineralization was measured by microscope and Image-J software.

\subsection{Real-time PCR}

The osteogenic induction of MC3T3-E1 cells using Ca/P-CDs was same as the above. When the induction was over, these MC3T3-E1 cells were collected by enzymatic digestion, and their total RNA was extracted by Trizol reagent. RT-PCR was conducted on the ViiA 7 RT-PCR System (Thermo Fisher Scientific) using the QuantiTect SYBR Green Kit (Qiagen, Quanta, France). The primer sequences of the related genes were listed in the Table 1. The expression of each related gene, including alkaline phosphatase (ALP), osteocalcin (OCN), and Runt-related transcription factor 2 (RUNX2), was normalized to the housekeeping gene ( $\beta$-actin), and fold differences were calculated using the comparative $C_{t}$ method. The osteogenic markers ALP, OCN and RUNX2, were analyzed.

Table 1

Primer sequences of real-time PCR reactions

\begin{tabular}{|llll|}
\hline Name & Primer & Sequence & Product \\
\hline ALP & Forward & 5'AACCCAGACACAAGCATTCC3' & 151 \\
\hline & Reverse & 5'GAGAGCGAAGGGTCAGTCAG3' & \\
\hline RUNX2 & Forward & 5'AGAGTCAGATTACAGATCCCAGG3' & 238 \\
\hline OCN & Foverse & 5'TGGCTCTTCTTACTGAGAGAGG3' & \\
\hline & Reverse & 5'GAGGACAGGGAGGATCAAGT3' & \\
\hline B-actin & Forward & 5'TCTTGGGTATGGAATCCTGTG3' & 81 \\
\hline & Reverse & 5'AGGTCTTTACGGATGTCAACG3' & \\
\hline
\end{tabular}

\subsection{In vivo animal study}

Mice calvarial defect model creation and Ca/P-CDs loaded hydrogel implantation

All animal procedures were conducted in accordance with the Management Rules of the Ministry of Health of the People's Republic of China and approved by the Institutional Animal Care and Use 
Committee of Jiangsu University (permit number: SYXK2018-053).

Four-week-old BALB/c mice were used (body weight $\sim 20 \mathrm{~g}$ ) for the experiments in vivo. Ten mice were randomly divided into two groups with 5 mice in each group. The brief surgical procedure was as follow: Firstly, the mice were anaesthetized with $10 \%$ chloral hydrate in normal saline by intraperitoneal injection at $3.5 \mathrm{ml}$ per $\mathrm{kg}$ body weight. Their fur on the skull surface was shaved off using an electric razor and this area was sterilized with lodine. Then, a $1.5 \mathrm{~cm}$ long longitudinal skin incision was made on the mouse scalp from the back of the eyes to the skull area using a sterile scalpel. The fascia was stripped laterally to expose the skull. Next, a $5 \mathrm{~mm}$ diameter defect was made using an electric drived low-speed (about $1500 \mathrm{rpm}$ ) trephine bur on one side of the parietal bone and cooled with normal saline during the whole process. Finally, $20 \mu \mathrm{L}$ of Ca/P-CDs loaded F127 hydrogel $(100 \mu \mathrm{g} / \mathrm{mL})$ was injected into the calvarial defect. After the surgery, the wound was sutured with simple interrupted suture and then the mice were placed separately and fed normally. CT images of the calvarial defect were acquired on a clinical 64-slice multidetector CT scanner (SOMATOM Emotion, Siemens, Bavaria, Munich, Germany). After 8 weeks, the major organs (heart, liver, spleen, kidneys, and lungs) were collected for conventional histocompability analysis.

\subsection{Statistical Analysis}

All experimental data were presented as mean \pm standard deviation (SD) and analyzed by one-way analysis of variance (ANOVA) or Tukey's multiple comparison tests between different treatments. $\mathrm{P}<0.05$ was considered statistically significant.

\section{Results And Discussion}

\subsection{Characterization of $\mathrm{Ca} / \mathrm{P}-\mathrm{CDs}$}

The successful preparation of Ca/P-CDs was performed by using calcium gluconate as calcium source and phosphoethanolamine as surface passivation agent based on Scheme 1. The morphology of synthesized $\mathrm{Ca} / \mathrm{P}-\mathrm{CD}$ s was observed using high-resolution transmission electron microscopy (HRTEM). As shown in Fig. 1A, the Ca/P-CD possessed discrete and quasi-spherical shape without apparent aggregation. The average diameter of $\mathrm{Ca} / \mathrm{P}-\mathrm{CD}$ s was approximately $2.4 \mathrm{~nm}$ that could be conducive to cross the physiological barrier and discharge from the body by the kidney. Figure 1B showed that there was no discernible lattice fringes, indicating the amorphous nature of $\mathrm{Ca} / \mathrm{P}-\mathrm{CD}$. XRD pattern was used to study the phase structure of $\mathrm{Ca} / \mathrm{P}-\mathrm{CD}$. As shown in Fig. $1 \mathrm{C}$, there was an strong and broad diffraction peak at $24.98^{\circ}$, which was similar to the characteristic peaks of graphite $\left(\{002\}\right.$ planes, $\left.2 \theta=26.5^{\circ}\right)$. Subsequently, we used Fourier infrared spectroscopy to study the chemical composition of Ca/P-CDs (Fig. 1D). FTIR spectroscopy of Ca/P-CDs showed the stretching vibration of $\mathrm{O}-\mathrm{H}$ at $3450 \mathrm{~cm}^{-1}, \mathrm{C}=\mathrm{O}$ at around $1650 \mathrm{~cm}^{-1}, \mathrm{C}-\mathrm{N} / \mathrm{C}-\mathrm{C}$ at around $1400 \mathrm{~cm}^{-1}, \mathrm{C}-\mathrm{O}$ at around $1100 \mathrm{~cm}^{-1}$, suggesting the abundant hydrophilic groups on the surfaces of $\mathrm{Ca} / \mathrm{P}-\mathrm{CD}$ s. 


\subsection{Chemical characterization of $\mathrm{Ca} / \mathrm{P}-\mathrm{CDs}$}

The surface chemical structure of $\mathrm{Ca} / \mathrm{P}$-CDs was further studied using X-ray photoelectron spectroscopy (XPS). As shown in Fig. 2A, there were five obvious peaks at 350,133, 284.0, 400.0, and 530.6eV, which indicated that the $\mathrm{Ca} / \mathrm{P}-\mathrm{CD}$ s were mainly composed of calcium, phosphorus, carbon, nitrogen, and oxygen atoms. The $\mathrm{N}_{1 \mathrm{~s}}$ spectrum displayed remarkable $\mathrm{N}-\mathrm{H}$ peaks at $399.9 \mathrm{eV}$ and $\mathrm{N}-\mathrm{C}$ peak at $399.3 \mathrm{eV}$, where the incorporation of $\mathrm{N}$ atoms could be used as auxochrome group to enhance the optical performance of Ca/P-CDs. The XPS spectrum showed the $\mathrm{P}_{2 p}$ peak at $133.60 \mathrm{eV}$ and $132.85 \mathrm{eV}, \mathrm{Ca}_{2 \mathrm{p}}$ peak at $350.70 \mathrm{eV}, 350.16 \mathrm{eV}, 347.30 \mathrm{eV}, 346.55 \mathrm{eV}$ and $346.85 \mathrm{eV}$ (Fig. 2E and 2F). It was verified that the Calcium and phosphorus elements were successfully doped into $\mathrm{Ca} / \mathrm{P}-\mathrm{CD}$, where both of the elements were essential for bone growth and differentiation.

\subsection{Optical characterization of $\mathrm{Ca} / \mathrm{P}-\mathrm{CDs}$}

The optical properties of $\mathrm{Ca} / \mathrm{P}-\mathrm{CD}$ s were characterized by photoluminescence (PL) spectrum and UV/Vis absorption spectrum. Figure $3 \mathrm{~A}$ showed there was no obvious absorption peak in an aqueous solution of $\mathrm{Ca} / \mathrm{P}-\mathrm{CD}$. Meanwhile, the aqueous solution was pale brown and transparent in daylight, but exhibited bright blue fluorescence under UV irradiation (inset, Fig. 3A). The Tyndall effect occurred when a bean of red laser gone through the aqueous solution, indicating that $\mathrm{Ca} / \mathrm{P}-\mathrm{CD}$ s had good dispersibility and colloid stability in water. The quantum yield of $\mathrm{Ca} / \mathrm{P}-\mathrm{CD}$ s was calculated at $11.3 \%$ using quinine bisulfate as a standard. As shown in Fig. 3A, the Ca/P-CDs displayed a broad range of emission wavelengths, resulting in preeminent multicolor fluorescent emission. In addition, the $\mathrm{PL}$ spectrum of $\mathrm{Ca} / \mathrm{P}-\mathrm{CD}$ s showed excitation-dependent emission manner, which was well similar to the conventional $\mathrm{CDs}^{[14,15]}{ }^{\text {. Moreover, }}$ the maximum excitation wavelength at $396 \mathrm{~nm}$ and the maximum emission wavelength at $480 \mathrm{~nm}$ further confirmed the blue fluorescence performance of Ca/P-CDs (Fig. 3C) .

\subsection{Biocompability of Ca/P-CDs}

Biocompatibility of nanomaterials is of great importance for biomedical applications. In this study, the cytocompatibility of $\mathrm{Ca}$ /P-CDs was evaluated by CCK-8 assay through incubation with VSMC and MC3T3-E1 cells. After $24 \mathrm{~h}$ incubation with $\mathrm{Ca} / \mathrm{P}-\mathrm{CD}$, the cell viability of VSMC and MC3T3-E1 cells was measured. As shown in Fig. 4A, it can be seen that cell viability of two groups did not show significant changes after treatment with Ca/P-CDs at $0,10,50,100,200,400,800 \mu \mathrm{g} / \mathrm{mL}$. Even at $800 \mu \mathrm{g} / \mathrm{mL}$, both of cell viability were still as high as $88 \%$ and $91 \%$, respectively.

Furthermore, the hemocompatibility of Ca/P-CDs was assessed by hemolytic assay. As shown in Fig. 4B, there was strong and sharp absorption peak at $541 \mathrm{~nm}$ in the positive control (DI Water group), indicating the presence of the hemolysis of red blood cells. Compared to the negative control (PBA group), the percentages of hemolysis were all less than $3 \%$ after treatment with Ca/P-CDs from 10 to $1000 \mu \mathrm{g} / \mathrm{mL}$, indicating that no significant hemolysis occurred. Taken together, these findings clearly suggested that the prepared Ca/P-CDs had negligible cytotoxicity and good biocompatibility. 


\subsection{Intracellular distribution of Ca/P-CDs after endocytosis}

Numerous studies have reported that the conventional CDs readily entered into cytoplasm in living cells due to their ultra small size. However, the intracellular localization of Ca/P-CDs after endocytosis was still unknown. In this study, three fluorescent probes (MitoTracker, ER-Tracker, or LysoTracker) were chosen to investigate the distribution of $\mathrm{Ca} / \mathrm{P}-\mathrm{CD}$ s at the organelle level. As shown in Fig. 5 , the presence of strong green fluoresce signal in three groups verified that $\mathrm{Ca} / \mathrm{P}-\mathrm{CD}$ s could be successfully internalized into cytoplasm after 6 hours of co-incubation. In addition, MC3T3-E1 cells had no obvious morphological changes. Furthermore, the red fluorescence from MitoTracker and ER-Tracker highly overlapped the green fluoresce from $\mathrm{Ca} / \mathrm{P}-\mathrm{CD}$, respectively. However, this fluorescence signal of colocalization for LysoTracker was relatively weak. According to this findings, we proposed that the $\mathrm{Ca} / \mathrm{P}-\mathrm{CD}$ s entering the cytoplasm were mainly distributed in the mitochondria and endoplasmic reticulum.

\subsection{Osteogenic induction of $\mathrm{Ca} / \mathrm{P}-\mathrm{CD}$ in vitro}

The function of osteogenic induction of $\mathrm{Ca} / \mathrm{P}-\mathrm{CD}$ s was explored by the RT-PCR and Alizarin red staining using MC3T3-E1 cells. Three different types of CDs was chosen as control treatments, including bare CDs (B-CDs), Calcium-doped CDs (Ca-CDs), Phosphorous-doped CDs (p-CDs). As shown in Fig. 6A and 6B, the mineralization of MC3T3-E1 osteoblast cell in the $\mathrm{Ca} / \mathrm{P}-\mathrm{CD}$ s treatment group was significantly higher than that of the other group. The function of $\mathrm{Ca} / \mathrm{P}-\mathrm{CD}$ s was further enhanced by the addition of the $\mathrm{G}$. In addition, the Ca-CDs group exhibited better osteogenic induction than the P-CDs group. Meanwhile, the expression of ALP, mature bone markers $(\mathrm{OCN})$ and osteogenic transcription factor (RUNX2) were significantly up-regulated under the same concentration of $\mathrm{Ca}-\mathrm{CDs}, \mathrm{Ca} / \mathrm{P}-\mathrm{CDs}, \mathrm{Ca} / \mathrm{P}-\mathrm{CDs}+\mathrm{GF}$ treatments, among which $\mathrm{Ca} / \mathrm{P}-\mathrm{CD}$ s and $\mathrm{Ca} / \mathrm{P}-\mathrm{CD}+\mathrm{GF}$ group has the highest expression. $\mathrm{Ca} / \mathrm{P}-\mathrm{CD}$ s could promote the expression of bone differentiation-related RNA to be 2-3 times higher than that of the control group (Fig. 6C-D). It can be proved that the Ca/P-CDs could effectively promote the expression of genes related to bone differentiation and promote development and maturity of bone differentiation.

\subsection{Osteogenic potential of $\mathrm{Ca} / \mathrm{P}-\mathrm{CD}$ in vivo}

Encouraged by the above results, we evaluated the ability of $\mathrm{Ca} / \mathrm{P}-\mathrm{CD}$ s to promote bone differentiation and development through in vivo experiments. In order to achieve administration in situ, F127 thermal sensitive hydrogel was chosen to load the Ca/P-CDs. After in situ injection, F127 hydrogel loading Ca/PCDs could form a gel quickly in the calvarial defect due to the body temperature rising. After 8 weeks of treatment, CT scans were performed to observe the healing of the skull defects. As shown in Fig. 7A, the calvarial defect of the mice was still observed after single F127 hydrogel treatment. However, the calvarial defect of the mice was gradually restored after Ca/P-CD s loaded F127 hydrogel treatment. The results verifed that $\mathrm{Ca} / \mathrm{P}-\mathrm{CD}$ s could effectively promote the repair of bone injury in vivo.

Besides, histological analysis of major organs were used to evaluated the potential long term biosafty of Ca/P-CDs. As shown in Fig. 7B, it showed no visible pathological changes in the lung, liver, spleen, kidney 
and heart after 8 weeks treatment with PBS, P-CDs, Ca-CDs, Ca/P-CDs. There was no obvious inflammation or quantum dot deposition in various organs. The literatures and our study had reported that CDs can be excreted out of the body through the kidneys, which reflected the good biological safety of CDs.

\section{Conclusion}

In this study, we have successfully prepared the Ca/P-CDs with osteogenic potential via introduction of calcium and phosphorus element by one-pot hydrothermal carbonization. The as-prepared Ca/P-CDs had uniform particle size distribution and good dispersibility in water. Furthermore, the Ca/P-CDs exhibited stable optical properties and favorable biocompatibility. After entering into the cytoplasm, the Ca/P-CDs could escape from the lysosome and mainly distributed in the mitochondria and ER. More importantly, these internalized $\mathrm{Ca} / \mathrm{P}-\mathrm{CD}$ s upregulated the expression of bone differentiation-related genes, including ALP, RUNX2 and OCN. Finally, the Ca/P-CDs promoted the development of osteoblasts in vitro and the repair of calvarial defect in vivo. In summary, the study can provide a novel alternative treatment for clinical bone injury repair and expand the applications of CDs in the biomedical fields.

\section{Declarations}

\section{Acknowledgement}

The research was supported by the Priority Academic Program Development of Jiangsu Higher Education Institutions (PAPD, 2018-87), Natural Science Foundation of Jiangsu Province (SBK2020022937), Key talents of young medicine in Jiangsu Province (QNRC2016444), Six talent peaks project in Jiangsu Province (No: WSN-281), Zhenjiang key research and development plan (SH2019002, SH2019060), Major natural science research projects of colleges and universities in jiangsu province (19KJA150004), Changzhou Sci\&Tech Program (Grant No .CJ20200108).

Conflicts of Interest: The authors declare no conflict of interest.

\section{References}

1. De Simone A, Evanitsky MN, Hayden L, Cox BD, Wang J, Tornini VA, Ou J, Chao A, Poss KD, Di Talia S (2021) Control of osteoblast regeneration by a train of Erk activity waves. Nature 590: 129-133

2. Dirckx N, Moorer MC, Clemens TL, Riddle RC (2019) The role of osteoblasts in energy homeostasis. Nat Rev Endocrinol 15: 651-665

3. Vaquette C, Mitchell J, Fernandez-Medina T, Kumar S, Ivanovski S. (2021) Resorbable additively manufactured scaffold imparts dimensional stability to extraskeletally regeneratedbone. Biomaterials 269: 120671

4. Adam S, Simon N, Steffen U, Andes FT, Scholtysek C, Müller DIH, Weidner D, Andreev D, Kleyer A, Culemann S, Hahn M. (2020) JAK inhibition increases bone mass in steady-state conditions and 
ameliorates pathological bone loss by stimulating osteoblast function. Sci Transl Med 12: 530

5. Okuchi Y, Reeves J, Ng SS, Doro DH, Junyent S, Liu KJ, El Haj AJ, Habib SJ. (2020) Wnt-modified materials mediate asymmetric stem cell division to direct human osteogenic tissue formation for bone repair. Nat Mater 1: 108-118

6. Pérez-Sayáns M, Somoza-Martín JM, Barros-Angueira F, et al. (2010) RANK/RANKL/OPG role in distraction osteogenesis. oral surg oral med oral pathol oral radiol endod 109: 679-686

7. Lu M, Chen H, Yuan B, Zhou Y, Min L, Xiao Z, Yang X, Zhu X, Tu C, Zhang X. (2020) The morphological effect of nanostructured hydroxyapatite coatings on the osteoinduction and osteogenic capacity of porous titanium. Nanoscale 12(47):24085-24099

8. Wang Z, Han T, Zhu H, Tang J, Guo Y, Jin Y, Wang Y, Chen G, Gu N, Wang C. (2021) Potential Osteoinductive Effects of Hydroxyapatite Nanoparticles on Mesenchymal Stem Cells by Endothelial Cell Interaction. Nanoscale Res Lett 16(1):67

9. Faggio G, Grillo R, Foti A, Agnello S, Messina F, Messina G. (2021) Micro- photoluminescence of Carbon Dots Deposited on Twisted Double-Layer Graphene Grown by Chemical Vapor Deposition. ACS Appl Mater Interfaces 13(6): 7324-7333

10. Li J, Yang S, Liu Z, Wang G, He P, Wei W, Yang M, Deng Y, Gu P, Xie X, Kang Z, Ding G, Zhou H, Fan X. (2021) Imaging Cellular Aerobic Glycolysis using Carbon Dots for Early Warning of Tumorigenesis. Adv Mater 33: 2005096

11. Chung S, Revia RA, Zhang M. (2021) Graphene Quantum Dots and Their Applications in Bioimaging, Biosensing, and Therapy. Adv Mater 33: 1904362

12. Liu J, Geng Y, Li D, Yao H, Huo Z, Li Y, Zhang K, Zhu S, Wei H, Xu W, Jiang J, Yang B. (2020) Deep Red Emissive Carbonized Polymer Dots with Unprecedented Narrow Full Width at Half Maximum. Adv Mater 32: 1906641

13. Zou S, Guo F, Wu L, Ju H, Sun M, Cai R, Xu L, Gong Y, Gong A, Zhang M, Du F. (2019) One-pot synthesis of cerium and praseodymium co-doped carbon quantum dots as enhanced antioxidant for hydroxyl radical scavenging. Nanotechnology 31(16): 165101

14. Zhang M, Zhao L, Du F, Wu Y, Cai R, Xu L, Jin H, Zou S, Gong A, Du F. (2019) Facile synthesis of cerium-doped carbon quantum dots as highly efficient antioxidant for free radical scavenging. Nanotechnology 30(32): 325101

15. Du F, Zhang L, Zhang L, Zhang M, Gong A, Tan Y, Miao J, Gong Y, Sun M, Ju H, Wu C, Zou S. (2016) Engineered gadolinium-doped carbon dots for magnetic resonance imaging-guided radiotherapy of tumors. Biomaterials 121: 109-120

16. Qin X, Liu J, Zhang Qing, Chen W, Zhong X, He J (2021) Synthesis of Yellow-Fluorescent Carbon Nano-dots by Microplasma for Imaging and Photocatalytic Inactivation of Cancer Cells. Nanoscale Res Lett 16(1): 14

17. Zhang L, Zhang J, Xu L, Zhuang Z, Liu J, Liu S, Wu Y, Gong A, Zhang M, Du F. (2021) NIR responsive tumor vaccine in situ for photothermal ablation and chemotherapy to trigger robust antitumor immune responses. J Nanobiotechnology 19(1): 142 
18. Sun W, Luo L, Feng Y, Qiu Y, Shi C, Meng S, Chen X, Chen H(2020) Gadolinium-Rose Bengal Coordination Polymer Nanodots for MR-/Fluorescence-Image-Guided Radiation and Photodynamic Therapy. Adv Mater 32(23): 2000377

19. Zhou Q, Gong N, Zhang D, Li J, Han X, Dou J, Huang J, Zhu K, Liang P, Liang XJ, Yu J, (2021) Mannose-Derived Carbon Dots Amplify Microwave Ablation-Induced Antitumor Immune Responses by Capturing and Transferring "Danger Signals" to Dendritic Cells, ACS Nano 15(2): 2920-2932 20. Miao S, Liang K, Zhu J, Yang B, Zhao D, Dongyuan Z, Kong B (2020) Hetero-atom-doped carbon dots: Doping strategies, properties and applications. Nano Today 33: 100879

\section{Figures}
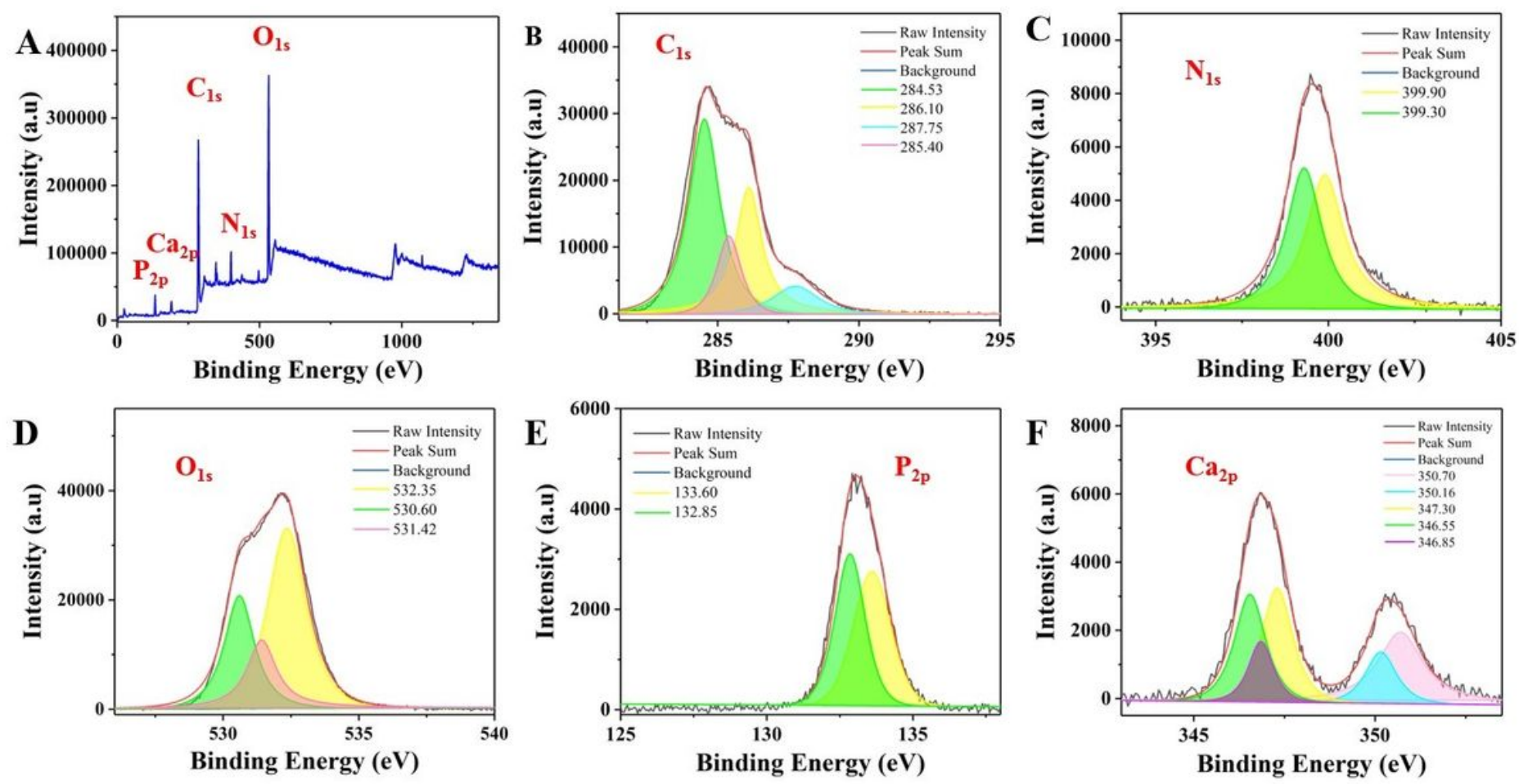

Figure 1

Characterization of the Ca/P-CDs. (A) TEM images, (B) HRTEM image, (C) XRD pattern, and (D) FTIR spectrum of the $\mathrm{Ca} / \mathrm{P}-\mathrm{CDs}$. 


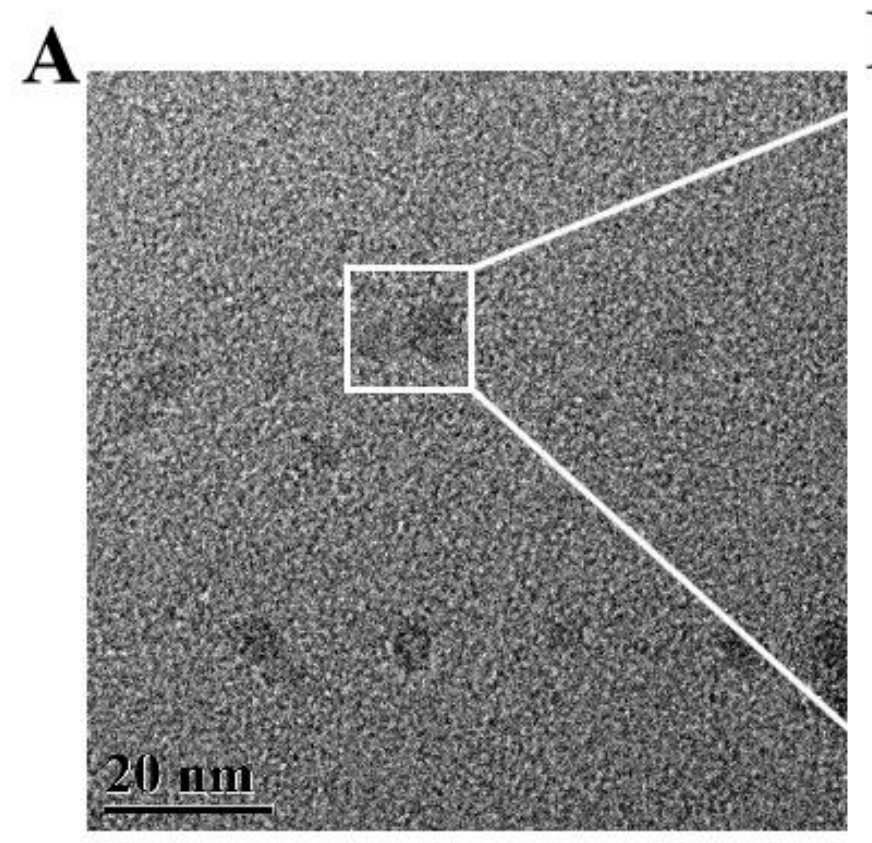

B
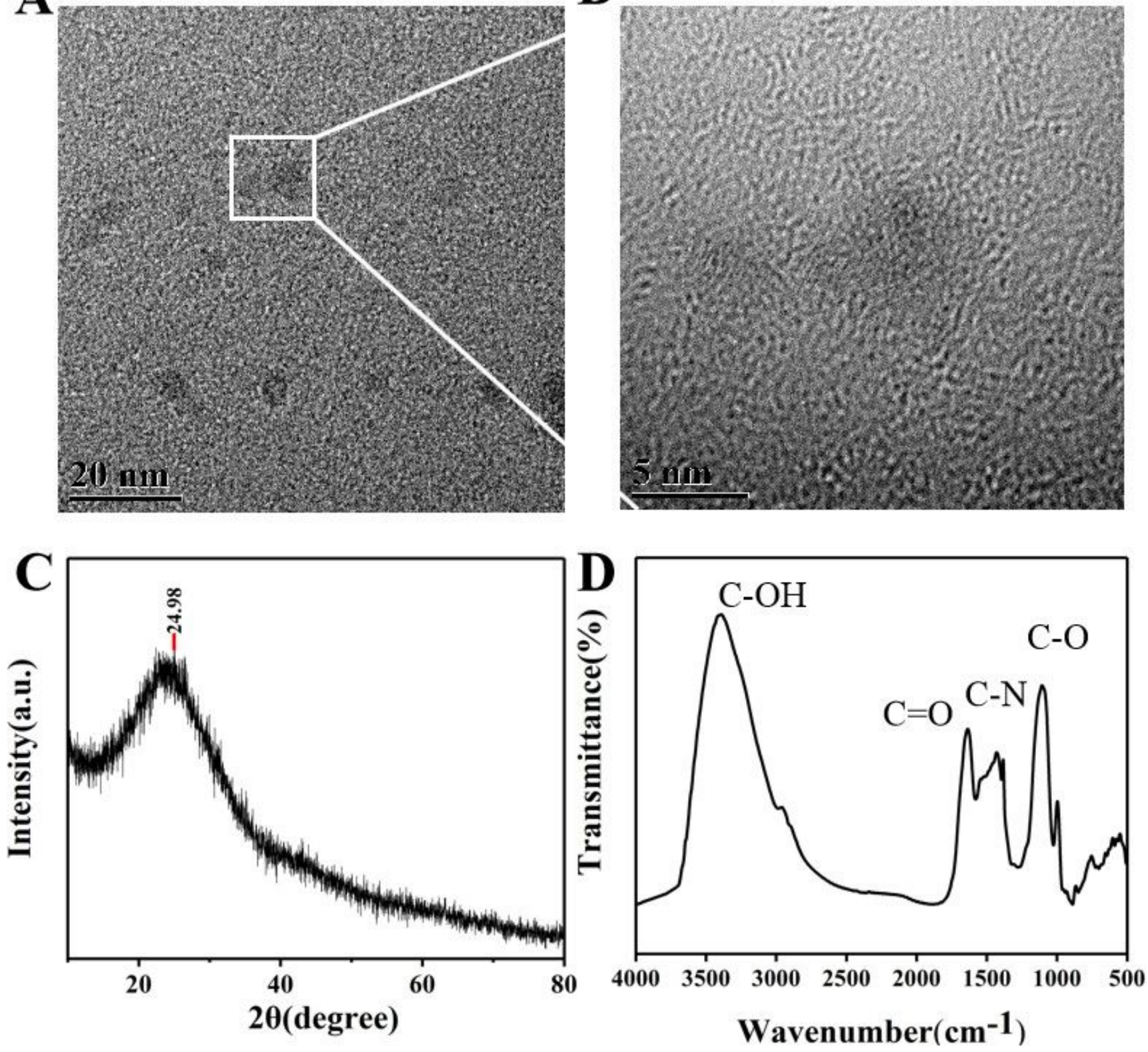

Figure 2

XPS spectra of the Ca/P-CDs. (A) Survey spectrum, (B) C1S spectrum, (C) N1S spectrum, (D) 01S spectrum, (E) P2p spectrum. (F) Ca2p spectrum. 

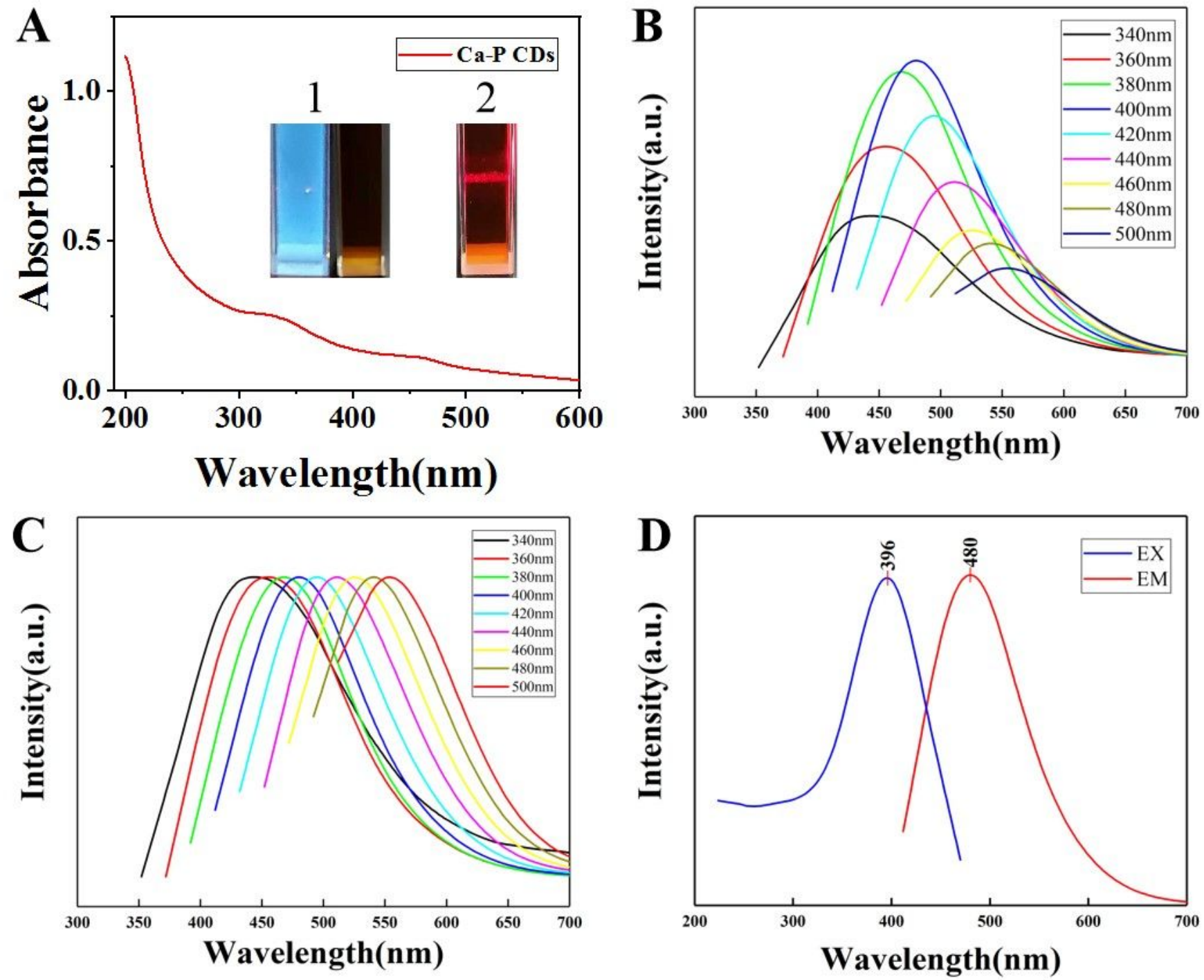

Figure 3

Optical characterization of Ca/P-CDs. (A) UV-vis absorption spectrum of $\mathrm{Ca} / \mathrm{P}-\mathrm{CD}$ s. Insert figure a represents the aqueous solution of $\mathrm{Ca} / \mathrm{P}-\mathrm{CD}$ s under sunlight and $\mathrm{UV}$ irradiation. Insert figure $\mathrm{b}$ represents a beam of red laser traverses the aqueous solution of $\mathrm{Ca} / \mathrm{P}-\mathrm{CD}$ s. (B) PL spectrum of Ca/P-CDs with different excitation wavelength from $340 \mathrm{~nm}$ to $420 \mathrm{~nm}$ respectively. (C) Normalized PL spectra of the $\mathrm{Ca} / \mathrm{P}-\mathrm{CDs}$. (D) Maximum emission spectrum of Ce-doped CQDs under maximum excitation wavelength. 

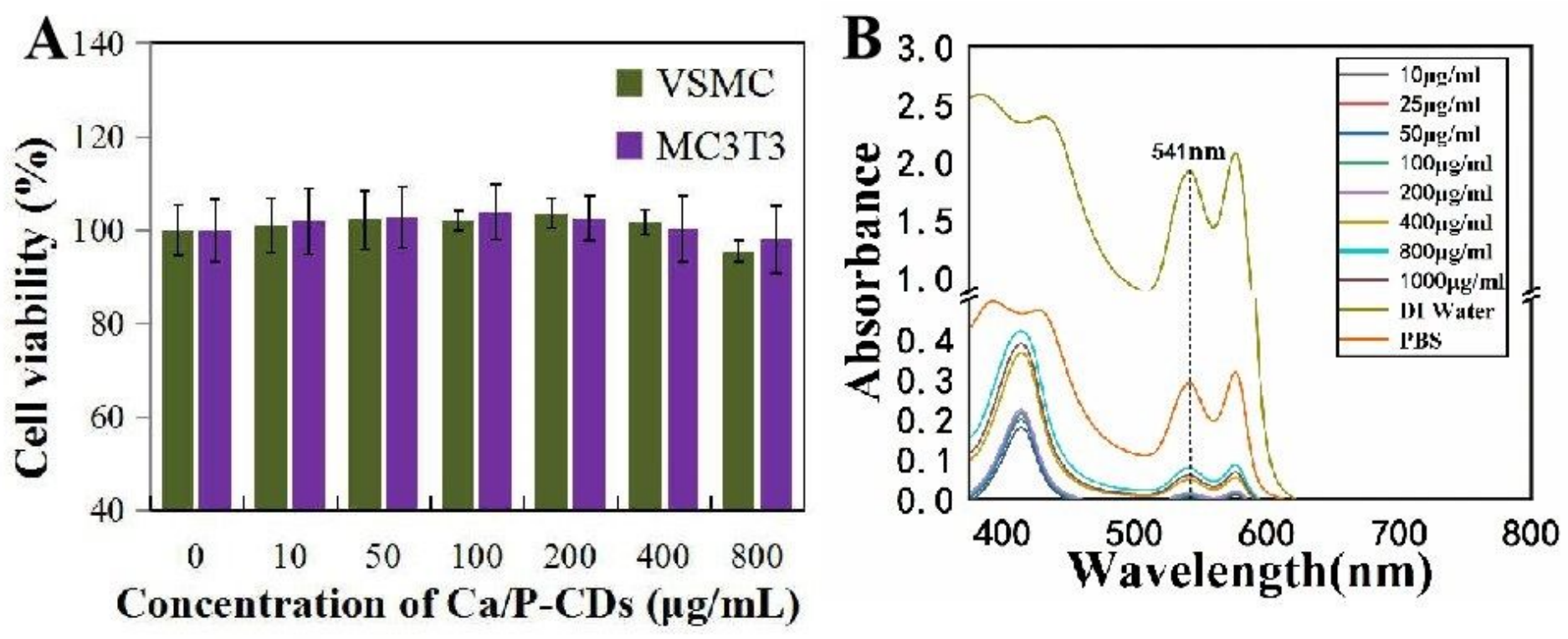

Figure 4

The effect of $\mathrm{Ca} / \mathrm{P}-\mathrm{CD}$ s with different concentration on cell viability $(A)$ and hemocompatibility $(B)$.

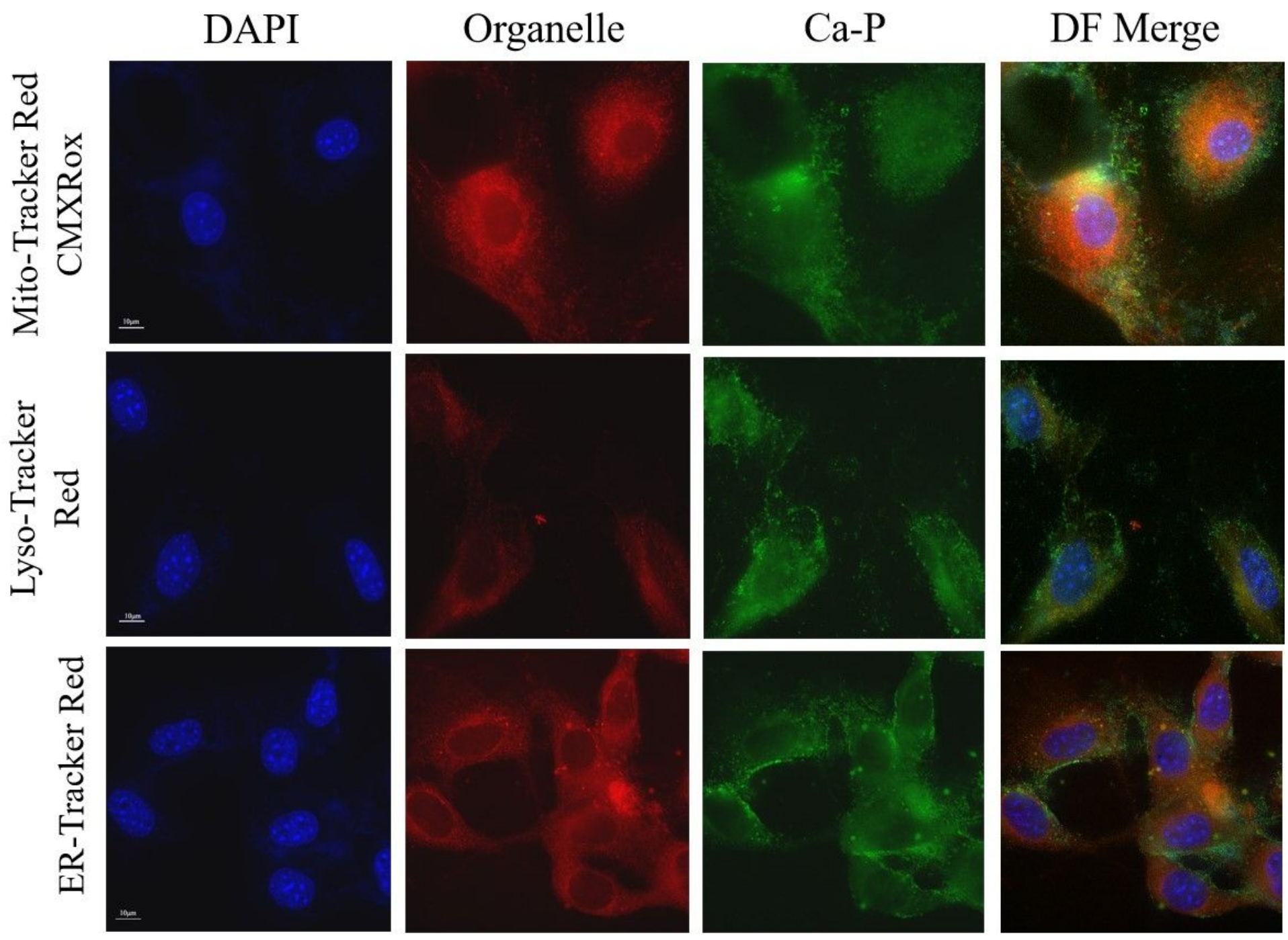


Figure 5

Fluorescent pictures of MC3T3-E1 incubated with Ca/P-CDs $(200 \mu \mathrm{g} / \mathrm{mL})$ using different organelle fluorescent probes.
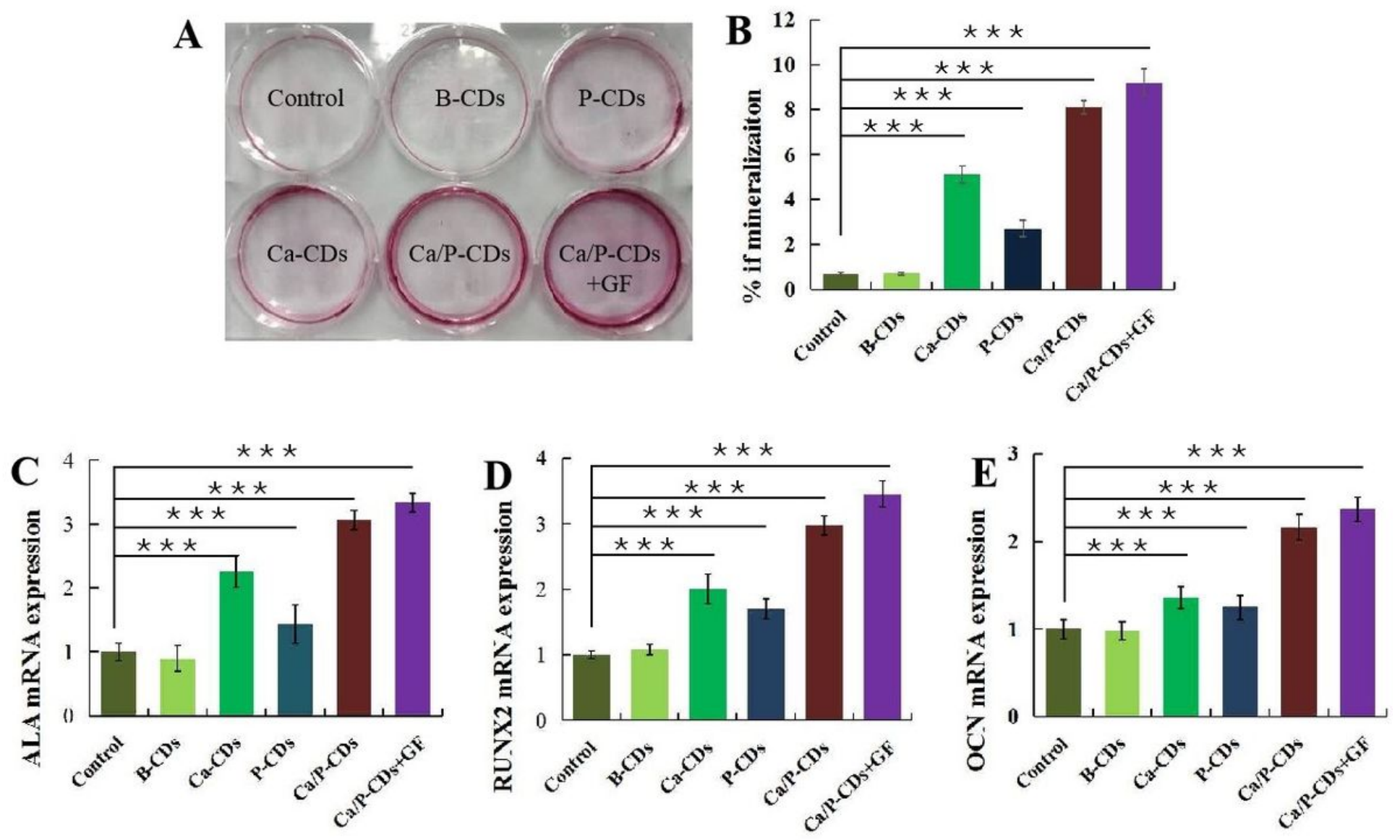

Figure 6

Evaluation of osteogenic potential of $\mathrm{Ca} / \mathrm{P}-\mathrm{CD}$ s in vitro. Alizarin red staining $(\mathrm{A})$ and quantitative analysis of MC3T3-E1 (B) after the Ca/P-CDs induction. Gene expression of ALP (C) , OCN (D), and RUNX2 (E) after the $\mathrm{Ca} / \mathrm{P}-\mathrm{CD}$ s induction. B-CDs: bare-carbon dots. Ca-CDs: Calcium-doped carbon dots. P-CDs: Phosphous doped carbon dots. GF: grwoth factor (rhBMP-2, $2 \mathrm{ng} / \mathrm{mL}$ ). 

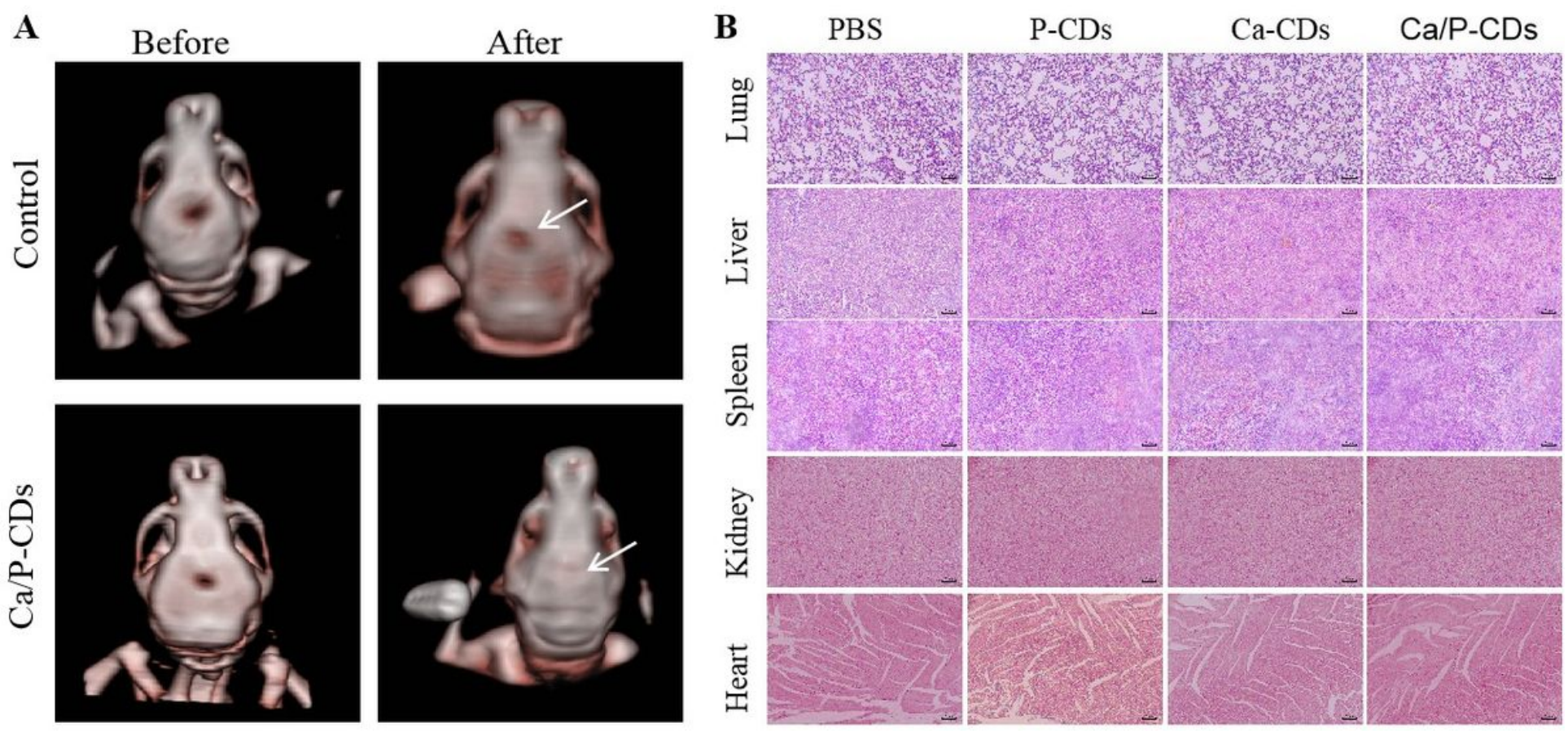

Figure 7

A, CT imagings of calvarial defect repair after $\mathrm{Ca} / \mathrm{P}-\mathrm{CD}$ s treatment. $\mathrm{B}, \mathrm{HE}$ analysis of major organs after 8 weeks of Ca/P-CDs treatment. The bars represent $20 \mu \mathrm{m}$.

\section{Supplementary Files}

This is a list of supplementary files associated with this preprint. Click to download.

- Scheme1.jpeg 